\title{
Research on the Model of Pressure Sensor used in PNS Based on Micro Jet 3DP
}

\author{
$\mathrm{Li} \mathrm{Na}^{1,2, *}$, Yang Jiquan ${ }^{1,2}$, Cheng Jihong ${ }^{1,2}$ and Qian Weixing ${ }^{2}$ \\ ${ }^{1}$ Jiangsu key laboratory of 3D printing equipment and manufacturing, Nanjing, 210042, China \\ ${ }^{2}$ School of Electrical and Automation Engineering, Nanjing Normal University, Nanjing, 210042, China
}

\begin{abstract}
In this paper, a low cost, printable pressure sensor model is presented. The pressure sensor could be used in personal navigation system which was designed based on micro jet fabrication structure. Micro jet printing and line patterning methods have been used to fabricate polymer resistors and field effect transistors on flexible substrates. A prototype sensor was designed, and the models of mechanical structure and digital fabrication was also given, fabricated and tested with standard experimental measurements. Results verify that the pressure sensitivity can be measured for both frequency and minimum power level difference in good performance. The dynamic performance of pressure sensor was also tested by micro jet printing on to flexible substrates including paper, with high resolution in just seconds. General structure of our proposed printable pressure sensor is detailed in the next section. Some resistors in pressure sensor were divided into two parts getting high sensitivity. The flexible spots and the elastic models for wearing substrate were given. In the experiment the flexibility, sensitivity and dynamic performance were tested which were in acceptable level.
\end{abstract}

Keywords: Three dimension print (3DP), Direct digital manufacturing (DDM), Printable sensor, Personal navigation system, Elastic model

\section{INTRODUCTION}

Research on MEMS pressure sensor has been concentrating in integration of sensing within the substrate for PNS (Personal Navigation System). The main goal is to get low cost, printable sensing capabilities. The benefit would be enhanced product quality, storage, transport and safety through ubiquitous sensing. A number of research groups have resulted in various printable sensor or actuator designs such as [1, 2] line based sensor (ii) pattern based (iii) R2R based etc. Fabrication progress makes it possible to produce more chipper sensor in large area with low requirement on substrate $[3,4]$.

Pressure is one of most important physical parameters of measurement device such as navigation device for directing, electronic products for sensing, undersea vehicles [3] avoiding collisions with moving object, and so on. So the pressure sensors are used in many fields to better our lives. It became smaller in volume and higher in resolution. What we want to do is to design the printable sensor on to soft substrate, like coats, shoes, paper and plastic. Moreover, the printable sensor is flexible and able to fit inside various structures [5, $6]$.

General structure of our proposed printable pressure sensor is detailed in the next section. Some resistors in pressure sensor were divided into two parts getting high sensitivity. The flexible spots and the elastic models for wearing substrate were given. In the experiment the flexibility, sensitivity and dynamic performance were tested.

\section{STRUCTURE OF THE PRINTABLE PRESSURE SENSOR}

In PNS (personal navigation system) the pressure sensor can give the height of the person wearing the device. The position on or under the bridge can be recognized by the device. The main component of the pressure sensor is the Wheatstone bridge architecture. The pressure on the thin film reflects on the change of the resistors. The PEDOT/PSS is used for micro jet printing to prototype the device [7-9], and the ink is polymer solution [10]. The circuit of pressure sensor is shown in Fig. (1).

The relationship between electric field and the stress can be described by the following equations:

$$
\begin{gathered}
S_{i}=S_{i j}^{E} \cdot T_{j}+d_{m i} \cdot E_{m} \\
D_{m}=d_{m j} \cdot T_{i}+\varepsilon_{m k}^{T} \cdot E_{k}
\end{gathered}
$$

Where d is the piezoelectric constant, and $S^{E}$ is the general parameters. Electromechanical coupling coefficient $\mathrm{K}$ is a comprehensive parameter reflecting the transfer ability of 


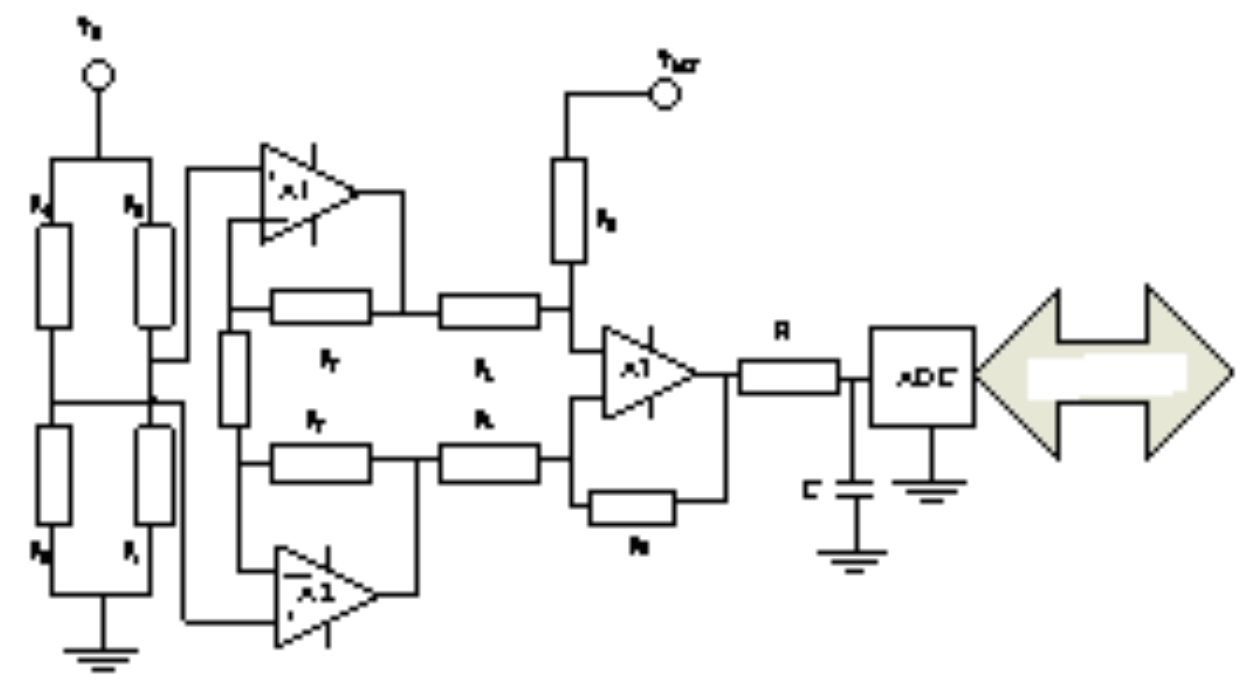

Fig. (1). Circuit of pressure sensor.

piezoelectric material between mechanical energy and electric energy. The definition of electromechanical coupling coefficient is as:

$$
K=\frac{U_{I}}{\sqrt{U_{M} U_{E}}}
$$

Where $U_{I}$ is the interaction density, $U_{M}$ is the elastic energy density, $U_{E}$ is dielectric energy density. The electric signal is:

$V_{3}=-h_{33} u_{m}\left(\frac{\sin ^{2}(k L)}{\cos (k L)}+(\cos (k L)-1)\right) e^{j \omega x}$

The gain of the circuit:

$$
\begin{aligned}
\frac{V_{o}}{V_{s}}= & \frac{R_{1}}{R_{1}+R_{2}}-\frac{R_{3}}{R_{3}+R_{4}} \\
& =\frac{R_{1} R_{3}-R_{2} R_{4}}{\left(R_{1}+R_{2}\right)\left(R_{3}+R_{4}\right)}
\end{aligned}
$$

Once the resistors change the output voltage changes, and the balance condition is: $R_{1} R_{3}=R_{2} R_{4}$, and the easy way to do is: $R_{1}=R_{2}=R_{3}=R_{4}=R_{0}$, and then $v_{o}=0$.

With the pressure changes, the resistor is changing to:

$$
R_{i, \text { new }}=\left(1+\alpha_{i}\right) R_{i, \text { old }}=\left(1+\alpha_{i}\right) R_{0}
$$

Where $\alpha_{1}=\alpha_{3}$ is the relative change in value, and with the same resistor value $\alpha_{1}=\alpha_{3}, \quad \alpha_{2}=\alpha_{4}$. Then

$$
\begin{aligned}
\frac{V_{o}}{V_{s}}= & \frac{\left(1+\alpha_{1}\right)^{2} R_{o}^{2}-\left(1+\alpha_{2}\right)^{2} R_{o}^{2}}{\left(2+\alpha_{1}+\alpha_{2}\right)^{2} R_{o}^{2}} \\
& \approx \frac{\left(\alpha_{1}-\alpha_{2}\right)}{2}
\end{aligned}
$$

Micro jet printing provides very good control of thickness and resolution of patterned active materials. The resistor in Wheatone could be divided into two parts that make the sensibility better. This can be seen as the sensitivity index. The structure in the thin film is designed as in Fig. (2). The pressure on the thin film produces the stress and the largest position is in the center of the film, which is called point CE. The longitudinal $l$ and transverse $t, \Delta R$ is one of the piezoelectric coefficient, $\Delta R$ is the pressure.

The ratio of the change in resistance $\Delta R$ to the resistance $\mathrm{R}$, called $\alpha$, of a resistor in a material with piezoresistive coefficients $\pi_{i}$ and stresses $\sigma_{i}$ is

$\alpha=\frac{\Delta R}{R}=\pi_{l} \sigma_{l}+\pi_{t} \sigma_{t}$

The subscripts $l$ and $t$ are the longitudinal and transverse stresses with respect to the resistor length axis.

Consider Fig. (2) which shows the placement of four resistors at the centers of the four edges of the surface of a square diaphragm with edge length $\mathrm{L}$ and thickness $\mathrm{H}$.

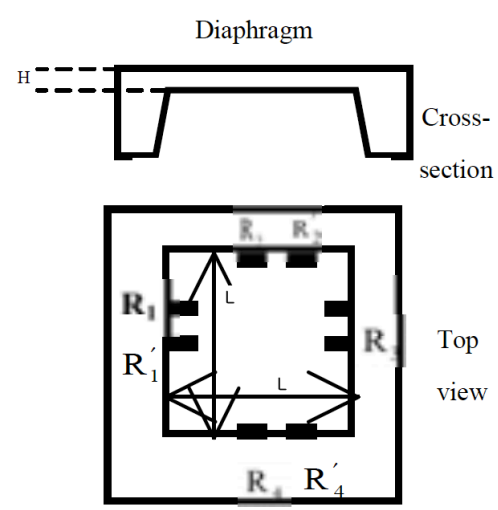

Fig. (2). Four resistors at the four edges of square diagram surfaces. 
When a pressure $\mathrm{P}$ is applied from above the diaphragm, the diaphragm experiences the stress and strain. As shown in [4] by finite-element methods (FEM), the stress (and strain) on the diaphragm is highest at the center of the edge, which we will call location DE, in the direction perpendicular to the edge:

$\sigma \perp, D E, F E M=0.243\left(\frac{L}{H}\right)^{2} P$

The strain in the parallel direction is

$\epsilon_{\|}=\frac{1}{E}\left(\sigma_{\|}-v \sigma_{\perp}\right)$

Because the four edges are fixed, there is no strain in the direction parallel to cur edge anywhere along the edge, i.e. $\epsilon_{\|}, e d g e=0$. Thus, the stress at the center of an edge in the direction parallel to the edge is

$\sigma_{\mathrm{P}}, D E, F E M=v \sigma_{\perp}, D E, F E M=0.023\left(\frac{L}{H}\right)^{2} P$.

For a real resistor of resistance $R$, the overall relative change is the weighted average relative change of its infinitesimal components, integrating lengthwise:

$\bar{\alpha}=\frac{1}{R} \int \alpha_{d R} d R=\frac{1}{R} \int_{s} \alpha_{d R}(s) \frac{\rho}{A(s)} d s$

Where $s$ is the distance along the length of the resistor, $\rho$ is the resistivity and $\mathrm{A}(\mathrm{s})$ is the cross-sectional area as a function of $s$.

The fabrication was layer by layer, as shown in Fig. (3). This is a structure of heterogeneous object. Considering that the material distribution of functionally graded material objects is very complicated, only a union method to deal with material distribution with multi-references.

\section{MODEL OF THE PRINTABLE PRESSURE SEN- SOR}

The model is designed for 3DP to fabricate the pressure sensor using in the PNS. The substrate is flexible for wearing.

\subsection{Elastic Mass Spot Model}

Micro jet printing techniques, have been gaining attention recently because of their unique features, such as simplicity of fabrication, compatibility with different substrates, feasibility of non-contact and no-mask patterning, low temperature processing and low cost. Micro jet printing is a familiar method used in nearly every office and household to transfer computer data to paper or transparencies. The same technology with a simple modification can be used to pattern solution processable polymers or nanoparticle inks on various substrates including glass, semiconductor materials, overhead transparencies and even paper. These methods are used in laboratory to fabricate electronic device in a flat substrate, but in most real applications the substrates are in different 3D shape, such as the shoes. The smart device is fab- ricated onto the uneven surface in different thickness. The 3D thin film should be used in such application. The model of $3 \mathrm{D}$ thin film from the $2 \mathrm{D}$ was given.

1) The flexible spots model

The plane thin film model was composed by mass spot $\mathrm{Q}$ and elastic line $\mathrm{L}$, as shown in Fig. (3) $Q=[M, P]$, $L=[F, q 0, q 1]$.

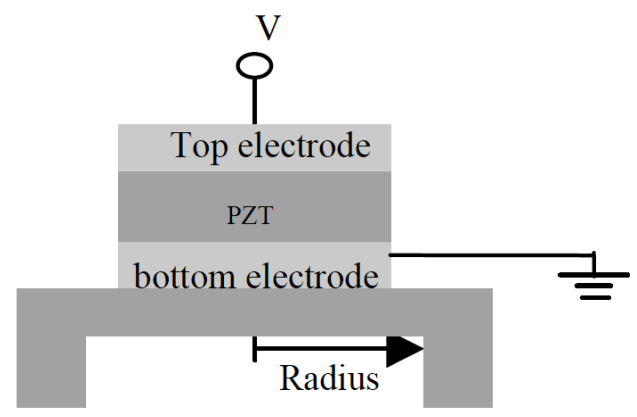

Fig. (3) The printable device structure.

In which the $\mathrm{M}$ is the material information, $\mathrm{P}$ is the position in the thin film, $\mathrm{F}$ is the elastic coefficient, and $\mathrm{q}_{0}$ and $\mathrm{q}_{1}$ are the spots the line $\mathrm{L}$ connects. Triangle and quadrangle can be composed using nearby points. Every spots has their sequence number in the film, which could be used in the fabrication with the triangle shape information.

\section{2) $2 \mathrm{D}$ to $3 \mathrm{D}$ mapping}

As given the area the $3 \mathrm{D}$ thin film is printed the $2 \mathrm{D}$ model is needed to be reflected to the $3 \mathrm{D}$ area. We used the method called STRETCH based on flexible spots. The main steps as follows:

(1) Given the maxim value of $Z$ in $3 D$ space, the center point of 2D space was assigned to it;

(2) Stretch the elastic lines of margins to cover the $3 \mathrm{D}$ shape. If failure the central point was changed and go back to (1);

(3) From the second point using the average length to stretch every point find the according position in 3D space, if failure the average length was changed and go back to (3);

The STRTCH model of thin film from 2D to $3 \mathrm{D}$ example is as in Fig. (4).

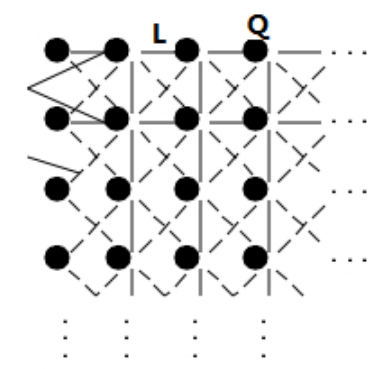

Fig. (4). Triangle grid of elastic mass point. 


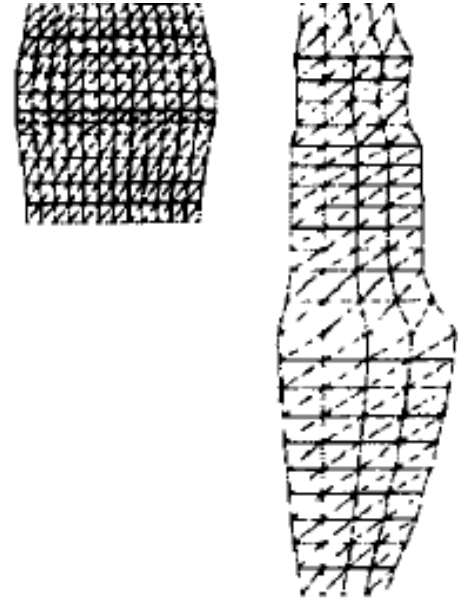

Fig. (5). Elastic model from 2D to 3D.

\subsection{Heterogeneous Material Model Representation}

To fabricate a prototype, it must first be represented in a CAD platform. If the concentration of each primary color binder is diluted in different degrees, when a color prototype is fabricated, a non-homogeneous or functionally graded one can be obtained. In general, a wider spectrum of binder concentration of a pixelm can be achieved by printing two binders with different concentrations in the appropriate proportion. To apply this concept for fabricating heterogeneous materials prototype, the proportion of the primary binders printed on different pixels of the object must be modeled so that varying binder concentration information can be obtained.

In the case of 3DP, only four primary binders are available (i.e, $\mathrm{n}=4$ ). yellow $(\mathrm{Y})$, and clear $(\mathrm{W})$ and their proportion vectors in the binder concentration simplex are denoted as $\mathbf{p}_{1}, \mathbf{p}_{2}, \mathbf{p}_{3}$, and $\mathbf{p}_{4}$ (corresponding to , $\mathbf{p}_{\mathrm{C}}, \mathbf{p}_{\mathrm{M}}, \mathbf{p}_{\mathrm{Y}}$, and $\mathbf{p}_{\mathrm{W}}$ ) respectively.

The corresponding sub-hyper-volume corresponding to $\mathbf{p}_{m}$ can be calculated as:

$$
M_{s u b-m 1}=\left(\begin{array}{l}
P_{m} \\
P_{2} \\
P_{3}
\end{array}\right), \quad M_{s u b-m 2}=\left(\begin{array}{l}
P_{m} \\
P_{3} \\
P_{1}
\end{array}\right), \quad M_{s u b-m 3}=\left(\begin{array}{l}
P_{m} \\
P_{1} \\
P_{2}
\end{array}\right), \quad M_{\text {Total }}=\left(\begin{array}{l}
P_{1} \\
P_{2} \\
P_{3}
\end{array}\right),
$$

In general, the individual component $\left(p_{m k}\right)$ of any point $p_{m}$ is equal to the corresponding $\alpha_{m k}$ if the vertices of the binder concentration simplex $\left(\mathrm{p}_{i}\right)$ are unit vectors and mutually orthogonal to each other, i.e.

$P_{i}=\left[\begin{array}{llllll}P_{i 1} & P_{i 2} & \mathrm{~L} & P_{i j} & \mathrm{~L} & P_{i m}\end{array}\right]$ and $P_{i j}= \begin{cases}0 & \text { ifi } \neq j \\ 1 & \text { ifi }=j\end{cases}$

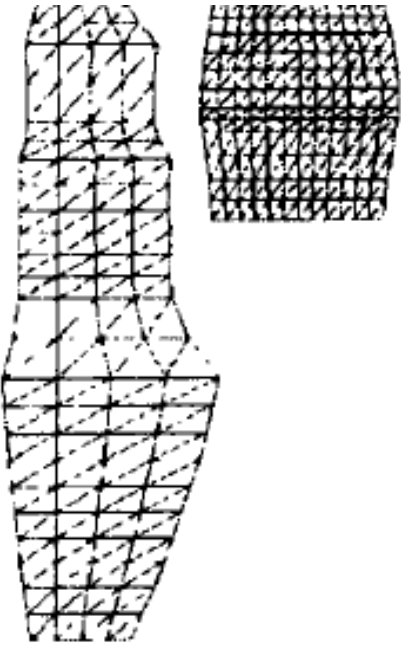

Three-dimensional printing is one of the DDM technologies that can be used for fabricating prototypes directly from CAD models in a layer-by-layer manner [4]. There was a large variety of 3DP processes introduced in the past decade. In 3DP, successive layers of prototype are printed until the whole prototype is fabricated. Post-treatment of the prototype (such as infiltrating the part with other materials for strengthening or producing the part with the specified infiltrated material) will also be performed. There are numerous applications of 3DP, including model verification, functional testing, form and fit checking, etc.

\section{FABRICATION BY MICRO JET 3DP}

The fabrication progress was simplified in Fig. (6). We detail eight strain-gauge resistors on a thin film in the pattern as in Fig. (7). The diaphragm is designed in odd symmetry to the center of the square, i.e. rotating $180^{\circ}$ or $90^{\circ}$ it is in the same structure, as shown in figure 2 . The two lines which are arranged in the center region change in the opposing direction. When the two resistors are arranged closer to the edges of the pattern they changed as well. All eight resistors have narrow lines $(10 \mu \mathrm{m}$ or so in a $2000 \mu \mathrm{m}$ film), which gets higher resistances, near the corresponding center areas where absolute strain is high. Since the ratio of widths to lengths is constant, the line resistance is scale-invariant.

Heterogeneous or FGM prototypes produced using multiple printer heads, different materials are printed on different parts of the layers of the prototypes. Both the inside and outside electric fields are controlled to produce the drops in different resolutions. In the first step the substrate was produced, and in the second step the diaphragm was printed on it.

Jetting resistor line based on Micro jetting design progress is for MEMS fabrication that is pressure sensors, which concentrate conductive line on the high-strain regions of the center in diaphragm without cross conduction lines. Another 

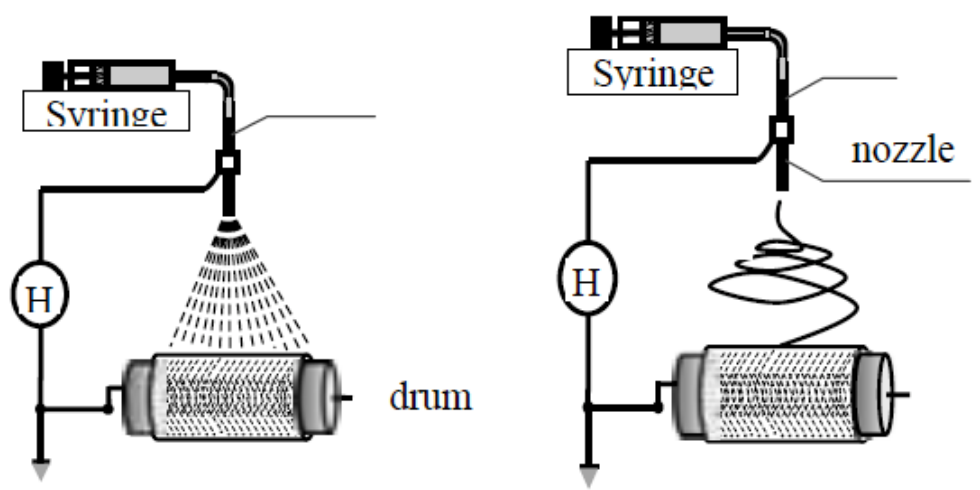

Fig. (6). Fabrication progress.
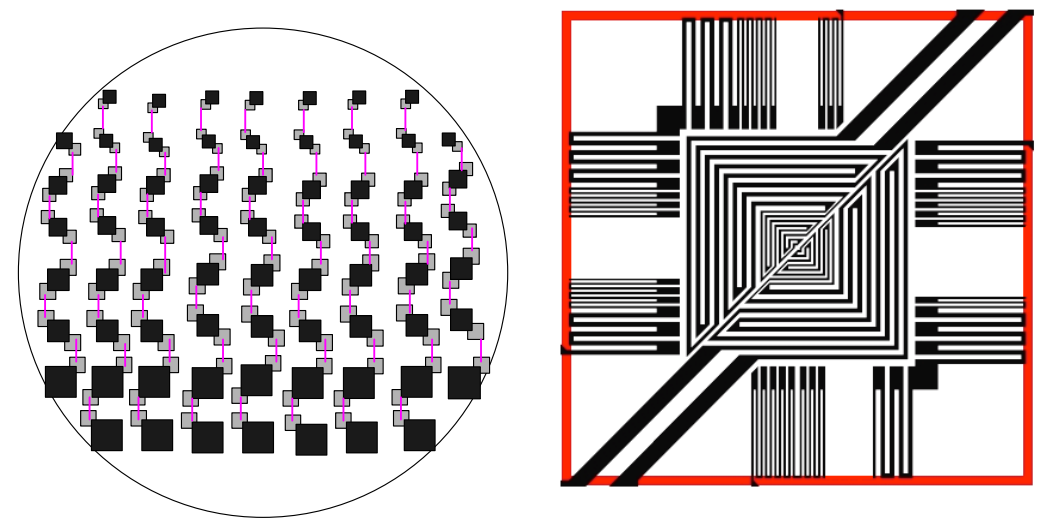

(a) Thin film structure (b) unit diagram

Fig. (7). The design of four strain-gauge resistors on a square diaphragm.

resistance is used here only for the loss in resolution. The high sensitivity was gotten in dynamic control. As shown in Fig. (7), the red square line indicates the boundaries of piece of diaphragm (nominally $2000 \mu \mathrm{m}$ or so), many kinds of micro structures can be molded using this structure.

The sensitivity and responsiveness are the main capability of pressure sensors. The two indexes are examined in the next section. The Jetlab platform of MicroFab was used in the examination, the progress was observed using CCD camera. The size could be controlled by different dynamic parameters of the piezoelectric jetting head. The three modes could be used to produce the drops, micro-drops and cone fluidic jetting.

\section{RESULTS}

Polymer-based resistors MEMS device have been fabricated using micro jet printing methods. Control the size and velocity of micro-drops to produce different line widths. The substrate is $3 \mathrm{D}$ surface which is sliced in software before fabrication for the printing. The elastic performance relates to the soft substrate, and are seen as flexibility. Also the several specifications were tested in the experiments.
A. Flexible Measurement: Under certain pressure, flexible performance is given as:

$w(r)=\frac{p_{0} a^{4}}{64 D}\left[1-\left(\frac{r}{a}\right)^{2}\right]^{2}$

When $\mathrm{r}=0$,

$w(r)=\frac{p_{0} a^{4}}{64 D}$

The diameter of the circuit was $400 \mu \mathrm{m}$, and the theoretical value of flexible was $22.8 \mu \mathrm{Nm}$. Center distance was $18.6 \mathrm{~nm} / \mathrm{kPa}$. In Fig. (8) the slop was $18.2 \mathrm{~nm} / \mathrm{kPa}$, and the error was $1 \%$ between the theoretical value and the measurement value.

B. Sensitivity: Thermal noise voltage is on the order of:

$V_{N} \approx \sqrt{4 k T R f}$

Where $k$ is Boltzmann's constant, $T$ is temperature, $R$ is resistance, and $f$ is the frequency. Thus, far $T=300 \mathrm{~K}, R=10$ 

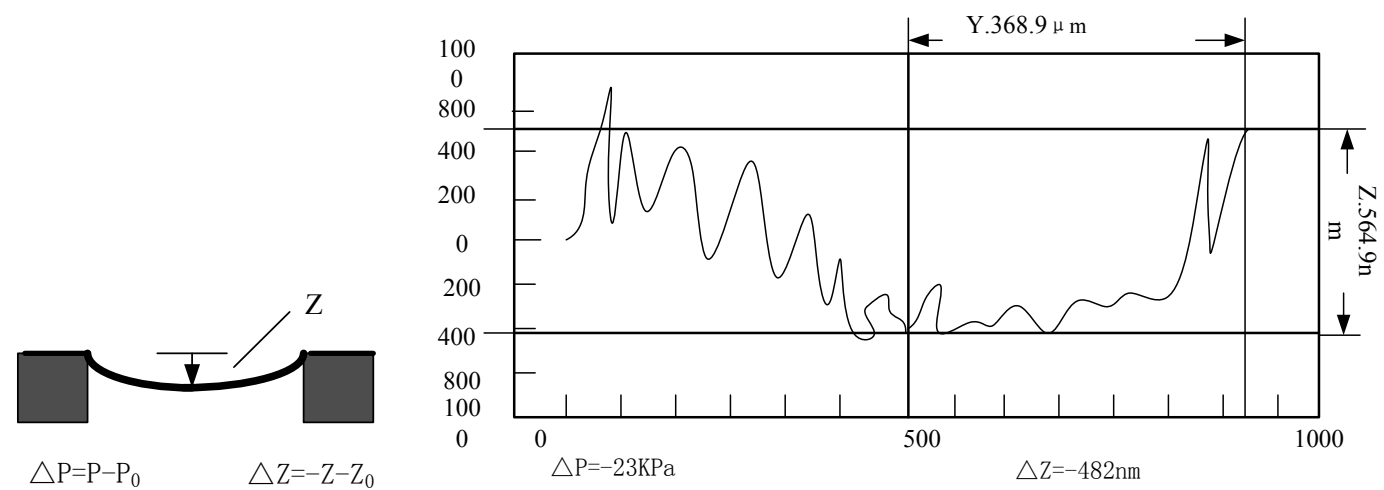

Fig. (8). Stiffness measurement.

$\mathrm{k} \Omega$, and a filtered bandwidth of $f=100 \mathrm{~Hz}$, the thermal noise voltage is approximately $0.13 \mu \mathrm{V}$. Therefore, the pressure resolution is on the order of $1.5 \mathrm{~Pa}$. Object detection and identification requires only $10 \mathrm{~Pa}$ resolutions, so the strain-gauge pressure sensor is sufficiently sensitive for navigation applications.

C. Dynamic: The expected performance parameters for the strain-gauge pressure sensors of various diaphragm lengths $L$, as discussed in earlier sections of this paper, are summarized in Table 1. All diaphragms have thickness $H=20$ $\mu m$ and all bridge source voltages are $\mathrm{Vs}=10 \mathrm{~V}$. The resistor performance is very important to the dielectric properties, inherent frequency and quality factor (Q). Capacity and energy consumption is related to the frequency, and changes largely. The loss factor is:

$\tan \delta=\omega \cdot C_{\text {ideal }} \cdot E S R$

$\varepsilon_{33}=\frac{t_{p} C_{\text {ideal }}}{A \varepsilon_{0}}$

Where $\omega$ is the angular frequency, $\varepsilon_{0}$ is the permittivity, $\varepsilon_{0}=8.85 \cdot 10^{-12}$. The results of the $\mathrm{n}$ times tests were researched. The range of the related capacitor: $750-890$, which is little. Loss factor was from $2.4 \%$ to $2.8 \%$, which is in reasonable extent. The quality factor and frequency were got given the value of $\mathrm{C} 1, \mathrm{R} 1, \mathrm{~L} 1, \mathrm{C} 0$.

Polymer-based resistors have been fabricated using line patterning and micro jet printing methods. The polymer resistors showed ohmic characteristics and as expected the resistance decreased with increase in the thickness of active material. The resistance of line patterned and micro jet printed PEDOT/PSS (Baytron P) has enhanced 900 and 350 time with ethylene glycol treatment. The promising performances of polymer resistors also the simplicity and low cost of their fabrication methods, would make it possible to integrate them into more complicated electronic structures to build disposable electronic devices on mechanically flexible substrates.

As shown in the experiments the flexibility, sensitivity and dynamic performance were tested which were in acceptable level.

\section{CONFLICT OF INTEREST}

The authors confirm that this article content has no conflicts of interest.

\section{ACKNOWLEDGEMENTS}

This work was financially supported by Natural Science Foundation of China (NSFC:61273243) and (NSFC:61304227).

\section{REFERENCES}

[1] W.K. Chiu, "Direct digital manufacturing of three-dimensional functionally graded material objects", Computer-Aided Design, vol. vol. 40, pp. 1080-1903, 2008.

[2] E.M. Amin, and N.C. Karmakar, "Development of a low cost printable humidity sensor for chipless RFID technology", International Conference on RFID -Technologies and Applications (RFID - TA), pp. 165-170, 2012.

[3] J. Virtanen, L. Ukkonen, T. Bjorninen, and A.Z. Elsherbeni, "Inkjet-printed humidity sensor for passive UHF RFID systems," IEEE Transactions on Instrumentation and Measurement, vol. 60, pp. 2768-2777, 2011.

[4] N.D. Sankır, Flexible Electronics: Materials and Device Fabrication, Dissertation submitted to the Faculty of Virginia Polytechnic Institute and State University, 2005.

[5] S.M.C. Hou, "Design and Fabrication of MEMS-array Pressure sensor Navigation Inspired by Lateral Line," Massachusetts Institute of Technology: USA, 2012.

[6] L. Yang, R. Zhang, D. Staiculescu, C.P. Wong, and M.M. Tentzeris, "A novel conformal RFID-enabled module utilizing printed antennas and carbon nanotubes for gas-detection applications", IEEE Antennas Propagation Letter, vol. 8, pp. 653-656, 2009. 
[7] F.M. Yaul, V. Bulovic, and J.H. Lang, "A flexible underwater pressure sensor array using a conductive elastomer strain gauge," Microelectromechanical Systems, vol. 21, pp. 897-907, 2012.

[8] C. Wang, K. Huang, D.T. W. Lin, W. Liao, H. Lin, and Y. Hu, "A flexible proximity sensor fully fabricated by inkjet printing", Sensors, vol. 10, pp. 5054-5062, 2010.
[9] N. Lenharta, K. Crowleyb, A.J. Killardc, M.R. Smythd, and A. Morrind, "Inkjet printable polyaniline-gold dispersions," Thin Solid Films, vol. 519, pp. 4351-4356, 2011.

[10] T. Unander, and H. E. Nilsson, "Characterization of printed moisture sensor in packaging surveillance applications", IEEE Sensors Journal, vol. 9, no. 8, pp. 922-928, Aug. 2009.

Received: September 16, 2014

Revised: December 23, 2014

Accepted: December 31, 2014

(C) Na et al.; Licensee Bentham Open.

This is an open access article licensed under the terms of the Creative Commons Attribution Non-Commercial License (http://creativecommons.org/licenses/by-nc/3.0/) which permits unrestricted, non-commercial use, distribution and reproduction in any medium, provided the work is properly cited.

\section{DISCLOSURE}

"Part of this article has already been published in Advanced Materials Research (Volumes 915-916), Chapter 14: Electronics Systems and Technologies, 1135-1139;Doi: 10.4028/www.scientific.net/AMR.915-916.1135”. 